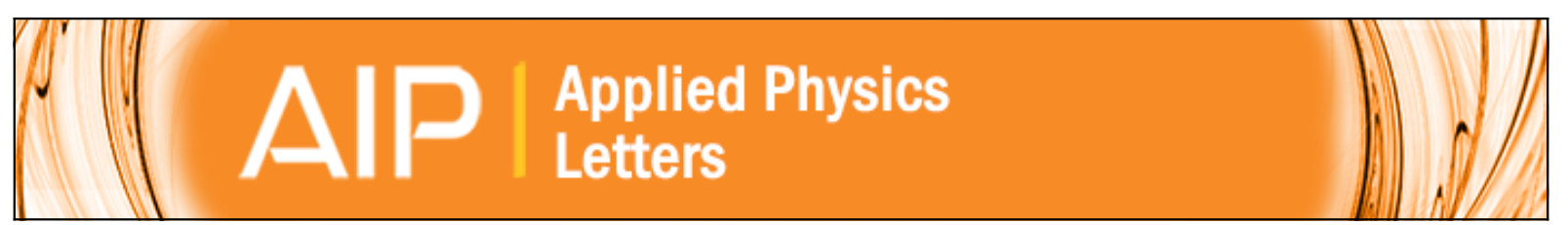

Beating of magnetic oscillations in a graphene device probed by quantum capacitance

M. Tahir and U. Schwingenschlögl

Citation: Applied Physics Letters 101, 013114 (2012); doi: 10.1063/1.4732796

View online: http://dx.doi.org/10.1063/1.4732796

View Table of Contents: http://scitation.aip.org/content/aip/journal/apl/101/1?ver=pdfcov

Published by the AIP Publishing 


\title{
Beating of magnetic oscillations in a graphene device probed by quantum capacitance
}

\author{
M. Tahir and U. Schwingenschlögla ${ }^{\text {a) }}$ \\ PSE Division, KAUST, Thuwal 23955-6900, Kingdom of Saudi Arabia
}

(Received 9 May 2012; accepted 18 June 2012; published online 5 July 2012)

\begin{abstract}
We report the quantum capacitance of a monolayer graphene device in an external perpendicular magnetic field including the effects of Rashba spin-orbit interaction (SOI). The SOI mixes the spin up and spin down states of neighbouring Landau levels into two (unequally spaced) energy branches. In order to investigate the role of the SOI for the electronic transport, we study the density of states to probe the quantum capacitance of monolayer graphene. SOI effects on the quantum magnetic oscillations (Shubnikov de Haas and de Hass-van Alphen) are deduced from the quantum capacitance. (C) 2012 American Institute of Physics. [http://dx.doi.org/10.1063/1.4732796]
\end{abstract}

Monolayer graphene is a gapless semiconductor with conical touching electron and hole bands. Since its discovery, this single atomic sheet of graphite has attracted much attention. ${ }^{1,2}$ The charge carriers obey a linear dispersion relation near the charge neutrality point (CNP, Dirac point), which endows the system with unique electronic properties. The different nature of the quasiparticles in graphene from conventional twodimensional electronic systems gives rise to unusual phenomena. ${ }^{3,4}$ Besides the fundamental interest in understanding the electronic properties of graphene, there are serious suggestions that it can serve as a building block for nanoelectronic devices. ${ }^{5-7}$ Quantum Hall measurements are one of the key tools providing evidence that the quasiparticles in graphene are chiral massless fermions, known as Dirac fermions. ${ }^{8-10}$

In recent years, lots of attention has been directed towards the role of the spin-orbit interaction $(\mathrm{SOI})^{11-16}$ in graphene based nanostructures because it introduces additional features. It has been predicted to induce a spin Hall effect. ${ }^{11}$ Along this line, the Rashba-type spin, which is larger than the intrinsic SOI, is also expected to differ fundamentally from that in the conventional two-dimensional electron gas. It plays a key role in determining the electronic transport properties of graphene. The occurrence of SOI in graphene is expected to greatly change the energy levels and, consequently the magnetic response. ${ }^{17}$ While SOI effects without magnetic field have received much attention, insight into the magnetic response of graphene in the presence of Rashba SOI is limited. In this context, we explore the effect of the SOI on the electronic properties of a graphene monolayer in an external perpendicular magnetic field.

Among the most important tools for studying the electronic properties of a material are capacitance measurements, which can be effectively used to probe the thermodynamic density of states of an electron system. Though the focus in graphene research has been on the transport properties, for the fundamental electronic properties as well as device physics it is important to study the capacitance-voltage (C-V) characteristics of the system. This approach recently has been realized for carbon nanotubes, graphene nanoribbons, and mono/bilayer graphene ${ }^{18-25}$ In general, attention is now being paid to electrostatic properties such as the quantum capacitance of graphene devices. Furthermore, to improve the performance of field effect transistors, ${ }^{26}$ usage of graphene as a channel material is creating a great deal of excitement. The present work complements earlier density of states and magnetocapacitance measurements ${ }^{18,22}$ of carbon based systems to determine the effects of the Rashba SOI.

We consider Dirac fermions in monolayer graphene (xy-plane) in the presence of an external perpendicular magnetic field. The two-dimensional Hamiltonian for Dirac fermions in a magnetic field is ${ }^{11,17}$

$$
H=v_{F}(\mathbf{p} \cdot \boldsymbol{\sigma})+\frac{1}{2} \lambda(\boldsymbol{\sigma} \times \mathbf{s})_{z} .
$$

Here, $\boldsymbol{\sigma}=\left(\sigma_{x}, \sigma_{y}\right)$ is the two-dimensional vector of Pauli matrices, $\mathbf{s}$ are the Pauli matrices for the real spin space, and $\mathbf{p}=\mathbf{p}+e \mathbf{A}$ is the two-dimensional canonical momentum with vector potential A. The spin-orbit coupling energy is $\lambda=13 \mathrm{meV}$ (Refs. 14 and 15) and $v_{F}$ denotes the Fermi velocity. We employ the Landau gauge and express the vector potential as $\mathbf{A}=(0, B x, 0)$. The electron (e) and hole (h) Landau levels energies are given by ${ }^{17}$

$$
\begin{gathered}
E_{0, s}^{e / h}=0, \\
E_{1,+}^{e / h}= \pm \sqrt{\lambda^{2}+2 \hbar^{2} \omega^{2}}, \quad E_{1,-}^{e / h}=0,
\end{gathered}
$$

and for $n \geq 2$,$$
\text { (1) }
$$
1

$$
E_{n, s}^{e / h}= \pm \sqrt{\frac{1}{2}\left[\lambda^{2}+2(2 n-1) \hbar^{2} \omega^{2}+s \sqrt{\lambda^{4}+4(2 n-1) \hbar^{2} \omega^{2} \lambda^{2}+\left(2 \hbar^{2} \omega^{2}\right)^{2}}\right]}
$$

\footnotetext{
a)udo.schwingenschlogl@kaust.edu.sa.
} 
where $s=+$ denotes spin up and $s=-$ denotes spin down. Moreover, $\omega=v_{F} \sqrt{(e B / \hbar)}$ is the cyclotron frequency and $n \in \mathbb{N}$.

We consider a gated graphene device in which the capacitor is formed between the gate and the graphene sheet. The quantum capacitance $C_{Q}$ of the device represents the charge response in the channel as the channel potential is varied, where $Q$ is the total charge on the graphene. In conventional devices, $C_{Q}$ is large and can be ignored, whereas in low dimensional devices, such as usual two-dimensional electron gases, graphene, and (in future) graphene spintronic devices, it is the dominant capacitive contribution and an important quantity for modelling the device. The quantum capacitance can be written as ${ }^{18-22}$

$$
C_{Q}=\frac{e \partial Q}{\partial \varepsilon_{F}}=\frac{e^{2} \partial n_{e}}{\partial \varepsilon_{F}}=e^{2} D_{T}(B),
$$

where $D_{T}(B)$ is the temperature dependent density of states at a finite magnetic field and $n_{e}$ is the carrier concentration. It is determined from the relation ${ }^{18}$

$$
D_{T}(B)=\frac{\partial n_{e}}{\partial \varepsilon_{F}}=\int_{0}^{\infty} d \varepsilon \frac{\partial f\left(\varepsilon-\varepsilon_{F}\right)}{\partial \varepsilon_{F}} D(\varepsilon) .
$$

Assuming a Gaussian broadening of the Landau levels, the density of states per unit area is given by

$$
D(\varepsilon)=\frac{2}{\pi l^{2}} \sum_{n=2}^{\infty} \sum_{s} \frac{1}{\Gamma \sqrt{2 \pi}} \exp \left[-\frac{\left(\varepsilon-E_{n, s}^{e / h}\right)^{2}}{2 \Gamma^{2}}\right],
$$

where $\Gamma$ is the Gaussian broadening. At the CNP, we have

$$
D\left(\varepsilon_{F}\right)=\frac{2}{\pi l^{2}} \sum_{n=0,1} \sum_{s} \frac{1}{\Gamma \sqrt{2 \pi}} \exp \left[-\frac{\left(\varepsilon_{F}-E_{n, s}^{e / h}\right)^{2}}{2 \Gamma^{2}}\right],
$$

where $\varepsilon_{F}$ is the Fermi energy. The results for $C_{Q}$ in Eqs. (5) to (8) reduce to the experimental findings ${ }^{18,22}$ in the limit of zero Rashba SOI.

In the numerical evaluation of $C_{Q}$, we assume a Gaussian broadening of the density of states, see Fig. 1, as a function of the Fermi energy (gate voltage). The following parameters are

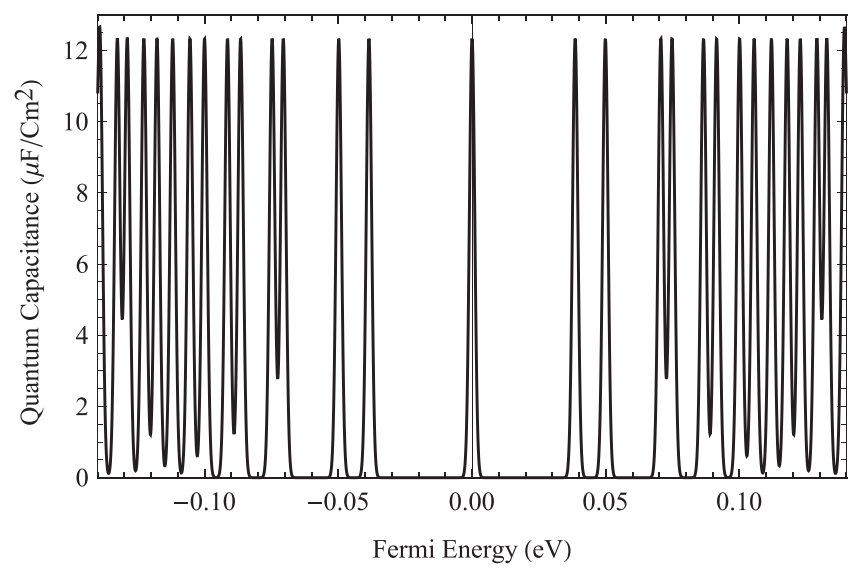

FIG. 1. Quantum capacitance as a function of the Fermi energy at zero temperature for a magnetic field of 2 Tesla. The Rashba SOI energy is $13 \mathrm{meV}$. employed: ${ }^{14,15,17,18,22} \Gamma=1 \mathrm{meV}, B=2$ Tesla, temperature $T=0, n_{e}=3 \times 10^{16} \mathrm{~m}^{-2}$, and $v_{F}=10^{6} \mathrm{~ms}^{-1}$. We do not find any splitting from the analysis of $C_{Q}$ at the CNP for $n=0$ and 1. It is important to note that the zero modes result in an additional four fold degeneracy at the CNP, unaffected by a change of the strength of the Rashba SOI. The splitting of the other levels ( $n \geq 2$ ) into two parts essentially is caused by the competition between the spin-up and spin-down contributions to the cyclotron energies. We find a maximum in $C_{Q}$ at the CNP, which is not affected by the Rashba SOI. All other Landau levels split into two parts with equal weights for the electron and hole contributions in the strong magnetic field regime. When the magnetic field is weak, we observe well resolved beating patterns of the magnetic oscillations (Shubnikov de Haas and de Haas-van Alphen) as a function of the perpendicular magnetic field, as shown in Fig. 2.

A well resolved beating pattern appears when the subband broadening is of the order of the Landau level separation. At high magnetic field, the effect of SOI weakens and the beating pattern is replaced by split magnetocapacitance peaks. The latter appears without SOI when the magnetic field becomes very strong. Accordingly, the conclusion can be drawn that the Rashba effect plays the main role in the formation of beating patterns in the magnetic oscillations in quantum capacitance measurements. Our numerical results are consistent with those given in Refs. 18 and 22, obtained in the limit of zero Rashba SOI.

Let us next discuss the experimentally observed regime of the Rashba SOI in electronic transport measurements. We estimate the cyclotron energy for $B=1$ Tesla as $\hbar \omega=36 \mathrm{meV}$. The level broadening with constant shift is $\Gamma=1 \mathrm{meV}$ and the Rashba SOI energy is $13 \mathrm{meV}$. Observation of a splitting of this energy requires several conditions: The temperature must be kept low and the system must be clean enough that disorder effects do not wash out the Rashba SOI effects. To attain this regime, temperature and disorder broadening must not reach the Rashba SOI energy. For our calculation, we have chosen a constant level width of $\Gamma=10$ Kelvin. In order to observe beating patterns of the magnetic oscillations, the magnetic field must be low.

The splitting of Landau levels is important for many physical quantities accessible in experiment, in addition to the quantum capacitance. As an example, we discuss the

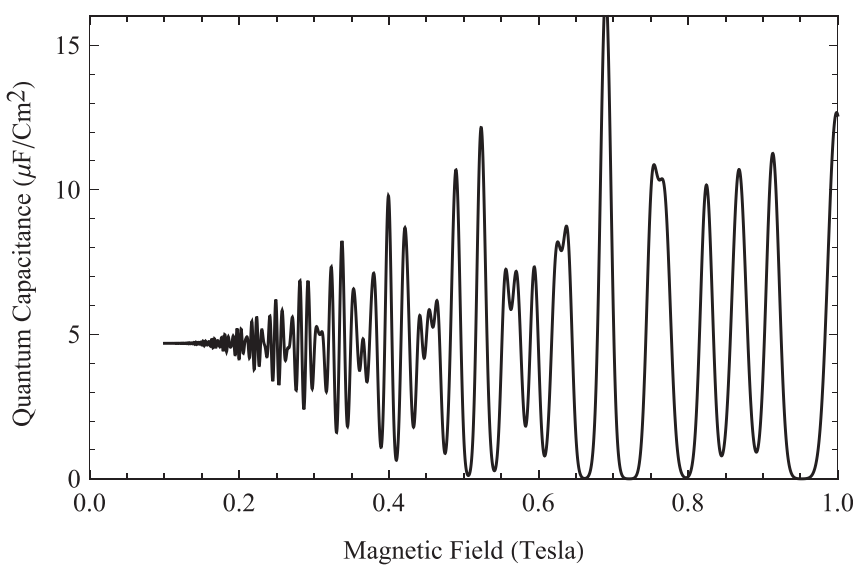

FIG. 2. Quantum capacitance as a function of the magnetic field at zero temperature for a Fermi energy of $100 \mathrm{meV}$. 
magnetoconductivity experiments in Ref. 27. The Boltzmann transport equation in case of short range scattering can be obtained from the Einstein relation in terms of the previously discussed density of states as $\sigma_{x x}=e^{2} v_{F}^{2} \tau D_{T}(B)$, where $\tau$ is the relaxation time.

In conclusion, we have investigated the effects of Rashba SOI on the quantum capacitance of a monolayer graphene device in a perpendicular magnetic field. We find that when the subband broadening is much smaller than the Landau level separation, the effect of the Rashba SOI on the capacitance manifests as a splitting of the magnetic oscillations peaks for the high magnetic field regime. For weak magnetic fields, with a level broadening comparable to the Landau level separation, a beating pattern appears in the quantum magnetocapacitance as a function of the magnetic field. Furthermore, no splitting is found at the CNP for $n=0$ and 1, while it is found for higher Landau levels $(n \geq 2)$ from the quantum capacitance. The developed theory is in good agreement with the available experimental data ${ }^{18,22}$ without Rashba SOI.

${ }^{1}$ K. S. Novoselov, A. K. Geim, S. V. Morozov, D. Jiang, M. I. Katsnelson, I. V. Grigorieva, S. V. Dubonos, and A. A. Firsov, Nature (London) 438, 197 (2005).

${ }^{2}$ Y. Zhang, Y. W. Tan, H. L. Stormer, and P. Kim, Nature (London) 438, 201 (2005).

${ }^{3}$ A. H. Castro Neto, F. Guinea, N. M. R. Peres, K. S. Novoselov, and A. K. Geim, Rev. Mod. Phys. 81, 109 (2009).

${ }^{4}$ D. S. L. Abergel, V. Apalkov, J. Berashevich, K. Ziegler, and T. Chakraborty, Adv. Phys. 59, 261 (2010).

${ }^{5}$ C. Berger, Z. Song, X. Li, X. Wu, N. Brown, C. Naud, D. Mayou, T. Li, J. Hass, A. N. Marchenkov, E. H. Conrad, P. N. First, and W. A. de Heer, Science 312, 1191 (2006).
${ }^{6}$ R. S. Deacon, K. C. Chuang, R. J. Nicholas, K. S. Novoselov, and A. K. Geim, Phys. Rev. B 76, 081406(R) (2007).

${ }^{7}$ S. Y. Zhou, G. H. Gweon, J. Graf, A. V. Fedorov, C. D. Spataru, R. D. Diehl, Y. Kopelevich, D. H. Lee, S. G. Louie, and A. Lanzara, Nat. Phys. 2, 595 (2006).

${ }^{8}$ S. G. Sharapov, V. P. Gusynin, and H. Beck, Phys. Rev. B 69, 075104 (2004).

${ }^{9}$ V. P. Gusynin and S. G. Sharapov, Phys. Rev. B 71, 125124 (2005).

${ }^{10}$ V. P. Gusynin and S. G. Sharapov, Phys. Rev. Lett. 95, 146801 (2005).

${ }^{11}$ C. L. Kane and E. J. Mele, Phys. Rev. Lett. 95, 226801 (2005).

${ }^{12}$ H. Min, J. E. Hill, N. A. Sinitsyn, B. R. Sahu, L. Kleinman, and A. H. MacDonald, Phys. Rev. B 74, 165310 (2006).

${ }^{13}$ D. Huertas-Hernando, F. Guinea, and A. Brataas, Phys. Rev. B 74, 155426 (2006).

${ }^{14}$ A. Varykhalov, J. Sanchez-Barriga, A. M. Shikin, C. Biswas, E. Vescovo, A. Rybkin, D. Marchenko, and O. Rader, Phys. Rev. Lett. 101, 157601 (2008).

${ }^{15}$ A. Varykhalov, D. Marchenko, M. R. Scholz, E. D. L. Rienks, T. K. Kim, G. Bihlmayer, J. Sánchez-Barriga, and O. Rader, Phys. Rev. Lett. 108, 066804 (2012).

${ }^{16}$ M. H. Liu, J. Bundesmann, and K. Richter, Phys. Rev. B 85, 085406 (2012).

${ }^{17}$ E. I. Rashba, Phys. Rev. B 79, 161409(R) (2009).

${ }^{18}$ L. A. Ponomarenko, R. Yang, R. V. Gorbachev, P. Blake, A. S. Mayorov, K. S. Novoselov, M. I. Katsnelson, and A. K. Geim, Phys. Rev. Lett. 105, 136801 (2010).

${ }^{19}$ S. Ilani, L. A. K. Donev, M. Kindermann, and P. L. McEuen, Nat. Phys. 2, 687 (2006).

${ }^{20}$ J. Guo, Y. Yoon, and Y. Ouyang, Nano Lett. 7, 1935 (2007).

${ }^{21}$ J. Xia, F. Chen, J. Li, and N. Tao, Nat. Nanotechnol. 4, 505 (2009).

${ }^{22}$ T. Yoshida and K. Oto, Physica E 42, 684 (2010).

${ }^{23}$ D. L. John, I. C. Castro, and D. L. Pulfrey, J. Appl. Phys. 96, 5180 (2004).

${ }^{24}$ F. Giannazzo, S. Sonde, V. Raineri, and E. Rimini, Nano Lett. 9, 23 (2009).

${ }^{25}$ H. Xu, Z. Zhang, and L. M. Peng, Appl. Phys. Lett. 98, 133122 (2011).

${ }^{26}$ T. Fang, A. Konar, H. L. Xing, and D. Jena, Appl. Phys. Lett. 91, 092109 (2007).

${ }^{27}$ S. Cho and M. S. Fuhrer, Phys. Rev. B 77, 081402(R) (2008). 